\title{
Central venous catheter-associated thromboembolism: still a frequent complication with no reliable predictive indicators in young children with diabetic ketoacidosis
}

\author{
Gary Woods ${ }^{1}$, Sarah O'Brien², Bryce Kerlin ${ }^{2}$, and Melissa Rose ${ }^{2}$ \\ ${ }^{1}$ Emory University School of Medicine \\ ${ }^{2}$ The Ohio State University College of Medicine
}

May 15, 2020

\begin{abstract}
Objective: Children presenting in diabetic ketoacidosis (DKA) may require a central venous catheter (CVC) to adequately manage their supportive care. These children are at increased risk of developing CVC-associated venous thromboembolism (VTE), but no predictive indicators have been identified to foretell which patients are at greatest risk. We analyzed demographic and laboratory data from children with DKA undergoing CVC placement to determine which patients may be at increased risk of CVC-associated VTE. Design: A retrospective chart review was conducted for patients aged 0-5 years admitted with DKA, CVC placement, and possible subsequent VTE development over ten years at a single institution. Reported demographic and laboratory variables were compared amongst patients that developed VTE and those that did not using Mann-Whitney rank sum tests. CVC-associated VTE incidence was also compared between children with DKA and all other patients undergoing CVC placement. Results: We identified 149 children with DKA, 17 underwent CVC placement, and 9/17 (52.9\%) developed CVC-associated VTE. Length of hospital stay was the only significant difference between those that developed VTE and those that did not. Also, the prevalence of catheter associated VTE in DKA (1.7\%) was significantly higher than that for CVC placement for any other reason $(\mathrm{p}<0.001)$. Conclusions: Careful consideration for CVC placement and minimizing duration of catheter use is suggested in this high risk population. Given the extremely high risk and lack of identifiable predictors, anticoagulation prophylaxis should be strongly considered for all young children with DKA requiring CVC placement.
\end{abstract}

\section{Introduction:}

Venous thromboembolism (VTE) is a rare but serious event in pediatrics that can cause significant morbidity and mortality. The estimated overall annual VTE incidence is approximately ten per 100000 children, with VTE diagnosed more commonly in hospitalized children, with a frequency of greater than 50 per 10000 [1-4]. Infants and adolescents are most often affected by VTE, and central venous catheters (CVC) have been estimated to be involved in up to $70 \%$ of pediatric VTE $[2,4]$. It has also been well-established that most pediatric VTE occur in patients with an underlying chronic medical condition or an acquired risk factor $[2,3]$.

Diabetes mellitus (DM) type 1 is a chronic medical condition characterized by immune-mediated pancreatic $\beta$-cell destruction, resulting in insulin deficiency. The incidence is increasing amongst all pediatric ages, but particularly in children under five years of age [5]. Diabetic ketoacidosis (DKA) is a serious complication of DM associated with ketosis, acidosis, and hyperglycemia [6]. Endothelial damage as well as hemostatic changes have been described in patients with DKA, including increased Factor VIII levels, increased fibrinogen, thrombocytosis, decreased protein $\mathrm{C}$ activity, and increased von Willebrand factor [7-9]. These hemostatic changes suggest enhanced coagulation potential and a prothrombotic state [10-14]. 
The management of a child with DKA can necessitate CVC placement to ensure appropriate therapeutic interventions are provided efficiently. CVC placement has been reported in up to $5 \%$ of children with DKA, but DKA management guidelines have recommended avoidance of CVC use implying the actual incidence of CVC placement for DKA management in children today could be less [15-17]. Children with DKA, particularly those under three years of age, undergoing CVC placement are at increased risk for VTE development (approximately 50\% VTE incidence), even more so than similarly aged children with CVC placement for a reason other than DKA [15, 18, 19]. Although young age has been associated with increased CVC-associated VTE in DKA, supporting evidence in the literature is weak. Also, other predictive indicators, including laboratory investigations, have not been evaluated to suggest which children with DKA undergoing CVC placement might be at higher risk for developing CVC-associated VTE.

In this report, DKA admissions to our facility over a ten year period were reviewed for patients aged less than five years old. Patients older than five years were not included because a preliminary analysis revealed that no CVC were placed in this population. We hypothesized that children with DKA would have a higher CVC-associated VTE incidence than all other patient groups and that young age would remain a risk factor for CVC-associated VTE in DKA.

\section{Materials and Methods:}

This study was reviewed and approved by the Institutional Review Board at Nationwide Children's Hospital prior to any data collection. Using the International Classification of Diseases (ICD-9)diagnosis codes for DKA (250.1x), billing database records were reviewed for children aged less than five years old between January 2002 and December 2011 with a DKA admission to Nationwide Children's Hospital in Columbus, $\mathrm{OH}$. DKA diagnosis was confirmed after chart review by laboratory results: venous $\mathrm{pH}<7.3$, hyperglycemia (blood glucose $>200 \mathrm{mg} / \mathrm{Dl}$ ), and ketonuria, as described by Woldorf et al [16]. This patient group was then cross-referenced for CVC placement (ICD-9 code 38.93 and 38.97) as well as VTE (ICD-9 code 453.2, 453.4x, 453.8x, and 453.9) diagnoses. All VTE codes were confirmed after chart review established that each case was diagnosed by Doppler ultrasonography and was CVC-associated.

Data were recorded for two patient groups: "DKA with CVC" and "DKA with CVC and VTE." Demographic and hospital course information was noted. Details of the CVC, including hospital day of placement, duration of use, type, size, location, continuous fluid infusion, and anticoagulation prophylaxis, were elucidated and compared between the two groups. Laboratory findings at DKA presentation were also reviewed to evaluate for predictive indicators for VTE in the setting of DKA with CVC. White blood cell count (WBC), hemoglobin, hematocrit, platelet count, blood urea nitrogen (BUN), creatinine, urine $\mathrm{pH}$, venous blood $\mathrm{pH}$, and hemoglobin $\mathrm{A} 1 \mathrm{C}$ were among those evaluated.

The patients that developed a VTE also had baseline coagulation studies (Prothrombin Time (PT), activated Partial Thromboplastin Time (aPTT)) and thrombophilia testing performed at the time of VTE diagnosis. Protein C activity, protein S activity, antithrombin activity, Prothrombin G20210A mutation, Factor V Leiden mutation, anticardiolipin antibodies, homocysteine levels, and quantitative D-dimer studies were investigated at the discretion of the hematologist.

Billing database records were also searched using the same ICD-9 codes for patients aged two months to five years of age for all CVC placements during our study period for any reason other than DKA. We elected to use two months as our lower age limit since our youngest patient with DKA and a CVC was two months old. This patient group was subsequently cross-referenced for VTE diagnoses up to three months after line placement and was compared to the DKA group for the incidence of CVC-associated VTE.

Descriptive statistics for patients with DKA and CVC are provided for all variables. Reported demographic and laboratory variables between groups were compared using Mann-Whitney rank sum tests. Fisher's exact test was used to compare the prevalence of CVC-associated VTEs between children with DKA and those without DKA. Statistical significance was determined by $\mathrm{p}<0.05$. Statistical analyses were performed using Statistical Analysis System software 9.3 (SAS Institute Inc., Cary, NC, USA). 


\section{Results:}

A total of 149 patients aged two months to five years were identified as having DKA between January 2002 and December 2011. Seventeen (11.4\%) of these patients underwent CVC placement for management of their DKA, of which nine $(52.9 \%)$ were subsequently diagnosed radiographically by Doppler ultrasonography with a CVC-associated VTE (Table 1). These nine events all occurred between days 3-5 of the admission for DKA. There were no VTE diagnoses in patients without CVC placement. Each CVC was placed on hospital day one either in the emergency room or intensive care unit and each patient received continuous fluid infusions for their DKA management for the duration of the CVC placement. None of the 17 identified patients had a documented family or personal history of thrombosis. The median age of the 17 patients with DKA undergoing CVC placement was 17 months, which was significantly younger than the 33-month median age of those for whom CVC placement was not necessary $(\mathrm{p}=<0.001)$. No CVC-associated VTE occurred after April 2007.

When comparing possible risk factors between patients with and without VTE (Table 2), the only statistically significant difference noted was that those with a VTE diagnosis had a longer median length of hospital stay ( 7 days) than those who did not $(4$ days $)(p=<0.003)$. The other demographic and laboratory measures did not significantly differ between these two groups.

All nine patients with CVC-associated VTE had anticoagulation management with enoxaparin or standard heparin with transition to enoxaparin. None of these patients had hemorrhagic complications and all were discharged on therapeutic enoxaparin doses.

Thrombophilia screening was performed on the nine patients diagnosed with a VTE at the discretion of the consulting hematologist. Protein $\mathrm{C}$ activity was normal in all patients except one, whose value was $12 \%$ at VTE diagnosis. One month into VTE treatment, protein C activity normalized at 91\%. Measured protein $\mathrm{S}$ activities were abnormal in two patients, aged 39 months and 12 months, whose values were $52 \%$ and $49 \%$ at VTE diagnosis with age specific lower limit of normal being $67 \%$ for both cases. These values were not reassessed post-treatment. Anticardiolipin IgG antibody testing was within the intermediate range at 16.8 GPL units in one patient (upper limit of normal is 15 GPL units). This value was not reassessed.

Over the 10-year study period, 3,038 CVCs were placed hospital wide for any reason other than DKA in patients aged two month to five years. Fifty two (1.7\%) developed a VTE within three months of line placement, 33 of which were diagnosed within one month. The prevalence of CVC-associated VTE in the 17 patients with DKA undergoing CVC placement (52.9\%) was significantly higher than that for CVC placement for any other reason $(\mathrm{p}<0.001)$.

\section{Discussion:}

The incidence of DKA amongst all pediatric age groups is rising, but especially in children less than five years of age [5]. In fact, DKA as the presentation of DM is much more common in children less than five years of age than any other age group, with up to $36 \%$ presenting with ketoacidosis [20, 21]. DKA management may necessitate CVC placement when obtaining peripheral access is difficult and infusion of medication and fluids is needed emergently. DKA is often associated with severe dehydration making it difficult to obtain IV access quickly, especially in children [22]. Adipose tissue distribution, smaller veins, and dehydration only enhances the difficulty [23].

Data from this study confirmed that children with DKA utilizing CVCs for disease management are at high risk for VTE development ( $~ 50 \%$ prevalence), and that children with DKA undergoing CVC placement are at a greater risk for developing CVC-associated VTE than those of a similar age group with CVC placed for any other reason. Our data also showed that children with DKA undergoing CVC placement are younger than those that do not (median age of 17 months vs. 33 months). Yet, assessment of both laboratory and demographic data amongst this patient population did not reveal any predictive indicators for VTE. In fact, the only significant difference between those with VTE and without VTE was length of hospital stay. This difference could be attributed to the acuity of the patients with VTE development. However, length of CVC 
use and degree of acidosis at presentation in the two patient groups was similar. A possible reason for the longer hospital stay was for VTE education and management.

At our institution from January 2002 through April 2007, there were 13 CVC placements for DKA management out of the 60 admitted patients. Specifically between February 2007 and April 2007, five of the seven patients admitted with DKA underwent CVC placement and three of these patients developed VTE. Only four of the 82 patients admitted for DKA underwent CVC placement after April 2007 and none had CVC-associated VTE. The decrease in CVC placement and CVC-associated VTE in this patient group is likely multifactorial. One patient had initial fluid resuscitation via an intraosseous tibial access at a referring hospital and had an external jugular CVC placed on arrival at our institution for continuing management. This patient's VTE risk may have been lessened by fluid resuscitation prior to CVC placement. Also, fewer CVC were placed for acute DKA management likely due to recent institutional experiences and increased awareness of the VTE risk in children with DKA undergoing CVC placement $[15,18,19]$. Two of the five patients that presented after April 2007 received continuous unfractionated heparin for VTE prophylaxis. Given the lack of identifiable predictive VTE indicators and very high risk ( $>50 \%$ in our population), anticoagulation prophylaxis should be strongly considered for all young children with DKA undergoing CVC placement.

The pathophysiology leading to a prothrombotic state in patients with DKA is not clearly understood. Possible mechanisms include that DKA is an acute worsening of a chronic hyperglycemic state. Both acute and chronic hyperglycemia are pro-inflammatory states, which has a known association with a prothrombotic state [24]. Studies have shown that critically ill non-diabetic children with hyperglycemia are at higher risk for VTE development than critically ill children without hyperglycemia [25]. DKA has also been associated with increased cortisol levels and growth hormone levels [24]. Unprovoked VTE have been described in patients with hypercortisolism or elevated growth hormone levels, suggesting an associated prothrombotic state [26]. Plasminogen has also been shown to be glycated in diabetes leading to decreased plasmin generation, impaired protein activity, and, subsequently, hypofibrinolysis [27]. More dedicated research is necessary to fully understand the mechanisms involved in the prothrombotic state associated with DM and DKA.

Using a retrospective chart review for data collection does have limitations. Thrombophilia testing was performed at the discretion of each provider, and thus was not always performed. Radiographic investigation for CVC-associated VTE was limited to patients that had VTE symptoms. Nevertheless, these limitations apply to both the DKA group and the control groups. Determining length of CVC placement was difficult in the non-DKA group using a retrospective chart review. We focused on VTE development within three months of CVC placement in an effort to capture any CVC-associated thrombus.

\section{Conclusions:}

Children aged less than five years with DKA have a higher risk of developing CVC-associated VTE than similarly aged children with CVC placement for any other reason. In our population, no demographic or laboratory markers were found to effectively predict which children with DKA would develop CVC-associated VTE. In accordance with published guidelines, CVC use in the management of pediatric DKA should be avoided if possible, but, if a CVC is placed, prophylaxis with standard or low molecular weight heparin should be strongly considered for all patients and the duration of CVC use should be limited by medical necessity.

\section{References:}

1. Boulet SL, Grosse SD, Thornburg CD, et al. Trends in venous thromboembolism-related hospitalizations, 1994-2009. Pediatrics2012; 130(4):e812-20

2. Andrew M, David M, Adams M, et al. Venous thromboembolic complications (VTE) in children: first analyses of the Canadian Registry of VTE.Blood 1994; 83(5):1251-1257

3. Raffini L, Huang YS, Witmer C, et al. Dramatic increase in venous thromboembolism in children's hospitals in the United States from 2001 to 2007. Pediatrics 2009; 124(4):1001-1008 
4. Setty BA, O'Brien SH, Kerlin BA. Pediatric venous thromboembolism in the United States: a tertiary care complication of chronic diseases.Pediatr Blood Cancer 2012; 59(2):258-264

5. Patterson CC, Dahlquist GG, Gyürüs E, et al. Incidence trends for childhood type 1 diabetes in Europe during 1989-2003 and predicted new cases 2005-20: a multicentre prospective registration study.Lancet 2009; 373(9680):2027-2033

6. White NH. Diabetic ketoacidosis in children. Endocrinol Metab Clin North Am 2000; 29(4):657-682

7. Carr ME. Diabetes mellitus: a hypercoagulable state. J Diabetes Complications 2001; 15(1):44-54

8. Carl GF, Hoffman WH, Passmore GG, et al. Diabetic ketoacidosis promotes a prothrombotic state. Endocr Res 2003; 29(1):73-82

9. Bilici M, Tavil B, Dogru O, et al. Diabetic ketoasidosis is associated with prothrombotic tendency in children. Pediatr Hematol Oncol2011; 28(5):418-424

10. Jenkins PV, Rawley O, Smith OP, et al. Elevated factor VIII levels and risk of venous thrombosis. $\mathrm{Br}$ J Haematol 2012;157:653-663.

11. Klovaite J, Nordestgaard BG, Tybjærg-Hansen A, et al. Elevated fibrinogen levels are associated with risk of pulmonary embolism, but not with deep venous thrombosis. Am J Respir Crit Care Med 2013;187(3):286-93.

12. Ho KM, Yip CB, Duff O. Reactive thrombocytosis and risk of subsequent venous thromboembolism: a cohort study. $J$ Thromb Haemost. 2012;10(9):1768-74.

13. Goldenberg NA and Manco-Johnson MJ. Protein C deficiency.Haemophilia 2008;14:1214-1221.

14. Flinterman LE, van Hylckama Vlieg A, Rosendaal FR, et al. Venous thrombosis of the upper extremity: effect of blood group and coagulation factor levels on risk.Br J Haematol 2010;149(1):118-23.

15. Worly JM, Fortenberry JD, Hansen I, et al. Deep venous thrombosis in children with diabetic ketoacidosis and femoral central venous catheters. Pediatrics 2004; 113(1 Pt 1):e57-60

16. Wolfsdorf J, Craig ME, Daneman D, et al. Diabetic ketoacidosis in children and adolescents with diabetes. Pediatric Diabetes2009;10(Suppl. 12): 118-133

17. Wolfsdorf J, Glaser N, and Sperling MA. Diabetic ketoacidosis in infants, children, and adolescents: a consensus statement from the American Diabetes Association. Diabetes Care 2006;29:5 1150-1159.

18. Gutierrez JA, Bagatell R, Samson MP, et al. Femoral central venous catheter-associated deep venous thrombosis in children with diabetic ketoacidosis. Crit Care Med 2003; 31(1):80-83

19. Davis J, Surendran T, Thompson S, et al. DKA, CVL and DVT. Increased risk of deep venous thrombosis in children with diabetic ketoacidosis and femoral central venous lines. Ir Med $J$ 2007; 100(1):344

20. Pinkey JH, Bingley PJ, Sawtell PA, et al. Presentation and progress of childhood diabetes mellitus: a prospective population-based study. The Bart's-Oxford study group. Diabetologica 1994; 37(1):70-4

21. Neu A, Willasch A, Ehehalt S, et al. Ketoacidosis at onset of type 1 diabetes mellitus in children frequency and clinical presentation.Pediatric Diabetes 2003; 4: 77-81

22. Dunger DB, Sperling MA, Acerini CL, et al. ESPE/LWPES consensus statement on diabetic ketoacidosis in children and adolescents. Arch Dis Child 2004; 89:188-94

23. Lillis KA, Jaffe DM. Prehospital intravenous access in children.Ann Emerg Med 1992; 21(12):1430-1434

24. Stentz FB, Umpierrez GE, Cuervo R, et al. Proinflammatory cytokines, markers of cardiovascular risks, oxidative stress, and lipid peroxidation in patients with hyperglycemic crises. Diabetes2004; 53(8):207986

25. Tala JA, Silva CT, Pemira S, et al. Blood glucose as a marker of venous thromboembolism in critically ill children. J Thromb Haemost 2014 Apr 8. doi: 10.1111/jth.12583

26. Squizzato A, Gerdes VEA, Ageno W, et al., The coagulation system in endocrine disorders: a narrative review. Intern Emerg Med 2007; 2(2):76-83

27. Ajjan RA, Gamlen T, Standeven KR, et al. Diabetes is associated with posttranslational modifications in plasminogen resulting in reduced plasmin generation and enzyme-specific activity. Blood 2013; 122(1):134-42

\section{Hosted file}

Table 1 DVT and DKA Demographics.docx available at https://authorea.com/users/322227/ 
articles/451307-central-venous-catheter-associated-thromboembolism-still-a-frequentcomplication-with-no-reliable-predictive-indicators-in-young-children-with-diabeticketoacidosis

\section{Hosted file}

Table 2 Demographics and laboratory values.docx available at https://authorea.com/users/ 322227/articles/451307-central-venous-catheter-associated-thromboembolism-still-afrequent-complication-with-no-reliable-predictive-indicators-in-young-children-withdiabetic-ketoacidosis 